\title{
Proteomics in non-human primates: utilizing RNA-Seq data to improve protein identification by mass spectrometry in vervet monkeys
}

J. Michael Proffitt ${ }^{1}$, Jeremy Glenn ${ }^{1}$, Anthony J. Cesnik², Avinash Jadhav ${ }^{1,6}$, Michael R. Shortreed², Lloyd M. Smith ${ }^{2,3}$, Kylie Kavanagh ${ }^{4}$, Laura A. Cox ${ }^{1,5}$ and Michael Olivier ${ }^{1,5,6^{*}}$

\begin{abstract}
Background: Shotgun proteomics utilizes a database search strategy to compare detected mass spectra to a library of theoretical spectra derived from reference genome information. As such, the robustness of proteomics results is contingent upon the completeness and accuracy of the gene annotation in the reference genome. For animal models of disease where genomic annotation is incomplete, such as non-human primates, proteogenomic methods can improve the detection of proteins by incorporating transcriptional data from RNA-Seq to improve proteomics search databases used for peptide spectral matching. Customized search databases derived from RNASeq data are capable of identifying unannotated genetic and splice variants while simultaneously reducing the number of comparisons to only those transcripts actively expressed in the tissue.

Results: We collected RNA-Seq and proteomic data from 10 vervet monkey liver samples and used the RNA-Seq data to curate sample-specific search databases which were analyzed in the program Morpheus. We compared these results against those from a search database generated from the reference vervet genome. A total of 284 previously unannotated splice junctions were predicted by the RNA-Seq data, 92 of which were confirmed by peptide spectral matches. More than half (53/92) of these unannotated splice variants had orthologs in other nonhuman primates, suggesting that failure to match these peptides in the reference analyses likely arose from incomplete gene model information. The sample-specific databases also identified 101 unique peptides containing single amino acid substitutions which were missed by the reference database. Because the sample-specific searches were restricted to actively expressed transcripts, the search databases were smaller, more computationally efficient, and identified more peptides at the empirically derived $1 \%$ false discovery rate.
\end{abstract}

Conclusion: Proteogenomic approaches are ideally suited to facilitate the discovery and annotation of proteins in less widely studies animal models such as non-human primates. We expect that these approaches will help to improve existing genome annotations of non-human primate species such as vervet.

Keywords: Proteogenomics, Proteomics, Liver, Vervet, RNA-Seq, Morpheus, Non-human primate, Galaxy-P

\footnotetext{
* Correspondence: molivier@wakehealth.edu

'Department of Genetics, Texas Biomedical Research Institute, San Antonio,

Texas, USA

${ }^{5}$ Southwest National Primate Research Center, Texas Biomedical Research

Institute, San Antonio, Texas, USA

Full list of author information is available at the end of the article
} 


\section{Background}

Shotgun proteomic approaches employ a database search strategy to compare experimentally observed mass spectra to an in silico-generated library of theoretical spectra derived from gene annotation information of the organism(s) being studied. The successful matching of peptides is thus predicated upon the accuracy of the search database being utilized to make these comparisons. The outcome of proteomics experiments is therefore driven by the quality and completeness of the genomic information of the organism being studied. Proteomic studies of genetically well-characterized species such as mice and humans benefit from robust proteomic search databases and extensive genome annotations which can account for known genetic variability such as splice variants and sequence variation altering the amino acid sequence of encoded proteins. However, protein identification of other research model organisms is limited by the quality of reference genome annotations.

Proteogenomic methods attempt to improve the search library limitations by leveraging information about gene transcription to guide the curation of search databases customized to the tissues of individual organisms. Several groups have demonstrated that transcriptional profiling using massively parallel sequencing approaches (RNA-Seq) improves the detection of peptides in proteomics experiments in a wide range of different species, ranging from microorganisms [1-3] and plants [4-7] to crustaceans [8], squids [9, 10], honey bees [11], chicken [12], ground squirrels [13], pig [14], and sheep [15]. The approach improves peptide assignment primarily in three important ways [16-18].

First, RNA-Seq data reveal sample-specific genetic sequences which may differ from the reference genome including nucleotide insertions, deletions, or substitutions. Single nucleotide polymorphisms (SNPs) comprise the majority of genomic variation within coding exons of genes, and these genetic variants are divided into two broad categories; SNPs which change a coding triplet but do not result in an amino acid substitution are referred to as synonymous SNPs, while variants which result in amino acid substitutions are called nonsynonymous SNPs (nsSNPs). The identification and inclusion of nsSNPs has the potential to improve the search database annotation because these changes can alter the chemical properties of the fragmented peptides. Failure to account for the resultant mass and/or charge change arising from the amino acid substitutions introduces ambiguity in peptide matching because, unlike nucleotide sequencing where the base order and fragment size can be directly inferred from the raw data, proteomic matching of peptides is based solely on the atomic mass and charge expected to be derived from enzymatically fragmented proteins.
Second, RNA-Seq reads can be used to identify splice junctions (SJs) which characterize mRNA isoforms absent from the reference gene model. SJs can result from genetic variation that alters how the spliceosome interacts with mRNAs, or SJs might arise as a result of alternative exon usage in tissue- or condition-specific contexts. RNA-Seq can also detect chimeric RNAs which arise from gene fusion events. Search databases that inadequately account for this isoform variability will fail to accurately identify their translated peptide products, and organisms with incomplete gene model information are therefore more susceptible to peptide misidentification.

Finally, RNA-Seq reads can be used to estimate transcript abundance. Knowledge of which mRNAs are expressed can inform how search databases can be trimmed to minimize multiple testing that occurs within peptide spectral matching algorithms. As with any iterative comparison process, the likelihood of misidentifying peptides increases with the total number of comparisons made. Experiments which incorporate proteomics results are vulnerable to Type I error inflation because multiple comparisons are made first at the peptide spectral matching identification stage, and then again in the context of the experimental condition (e.g. identifying protein abundance differences between case and control groups).

Based on this summary, it is evident that samplespecific search databases derived from RNA-Seq analyses from the same tissue sample as the proteomics data provide an improved search database, as it will include those peptides mostly likely to be found within the tissue being sampled, while excluding records derived from extraneous genomic information. This approach becomes even more valuable for analyses in samples from species with poorly annotated genomes [19], for reasons we discuss below.

Several bioinformatics pipelines have been described which facilitate the conversion of RNA-Seq reads into customized peptide databases which can be used to search mass spectrometry (MS) data. A well-established approach, developed and described by Sheynkman et al. [16], leverages the web interface of the Galaxy bioinformatics project [20] to facilitate the coordination of independent bioinformatics tools into a functional, user-generated proteogenomic workflow. As part of our analysis, we specifically implemented the proteogenomics approach using the analysis program Morpheus [21] which is computationally less demanding than other programs, and effectively calculates empirical false discovery rates (FDR) for peptide matches. We utilized this approach to determine whether RNA-Seq data from vervet monkey liver samples, a non-human primate 
without a well-characterized and annotated genome, improves the detection of peptides in vervet liver proteomic data.

While several non-human primate animal models for disease have been extensively used for decades, only recently have the genomes of these organisms begun to be characterized. The African green monkey, or vervet monkey (Chlorocebus aethiops sabeus), is one such example. The vervet monkey has long been an important model in AIDS research, as vervets are natural carriers of SIV yet display no symptoms of illness upon infection [22, 23]. More recently, vervets have provided insight into neurologic $[24,25]$ and metabolic diseases [26-29]. With the recent release of the first vervet genome [30], this animal model is ideally suited to benefit from the expansion of its genome annotation that proteogenomic approaches can provide. In this paper, we utilize matched RNA-Seq and MS data from vervet liver samples to characterize the vervet liver proteome and demonstrate that proteogenomic methods improve the detection of peptides otherwise missed by search databases constructed from the current reference vervet genome annotation.

\section{Methods}

\section{Sample collection}

All experimental procedures involving animals were approved and complied with the guidelines of the Institutional Animal Care and Use Committee of Wake Forest University Health Sciences and conducted in AAALAC approved facilities. All animals included in this study were female vervet/African green monkeys (Chlorocebus aethiops sabaeus) from the Vervet Research Colony (VRC) at Wake Forest School of Medicine. All monkeys were US-colony born within the VRC, which is a multi-generational, pedigreed, and genotyped colony originally founded in 1975 by the University of California Los Angeles, with 57 animals imported from St. Kitts and Nevis. In early 2008, the VRC was transferred to Wake Forest School of Medicine and remains a continuously NIH-supported national research resource. To obtain the samples reported here, 10 vervet monkeys were sedated with ketamine $(15 \mathrm{mg} /$ $\mathrm{kg}$ intramuscularly), intubated, and anesthetized using isoflurane to facilitate the surgical retrieval of liver tissue via laparotomy. Liver tissue was immediately frozen in liquid nitrogen and stored at $-80 \mathrm{C}$ until analysis.

\section{RNA-Seq}

Total RNA was extracted from vervet monkey livers using the Zymo Direct-zol ${ }^{\text {ma }}$ kit (Zymo Research, R2070) and each sample was subsequently quantified by Qubit assay (Thermo Fisher, Q32852). RNA-Seq libraries were prepared from $500 \mathrm{ng}$ of total RNA according to the Illumina TruSeq stranded mRNA protocol (Illumina,
RS-122-2101), which specifically retains polyadenylated mRNAs through the use of oligo dT coated magnetic beads. Sequencing library concentrations were quantified using the KAPA library quantification kit (Kapa Biosystems, KK4824). Clusters were generated by cBot (Illumina), and $2 \times 100$ base paired-end sequencing libraries were sequenced using the Illumina HiSeq 2500 with v3 sequencing reagents (Illumina, FC-401-3001).

\section{Conversion of RNA-Seq data to customized peptide databases in galaxy-P}

Methods for converting the RNA-Seq reads into searchable protein databases have been extensively described previously [16, 31, 32]. We adapted these approaches within Galaxy-P to create sample-specific search databases for each of the 10 vervet monkey liver samples, using the reference vervet monkey genome (ChlSab1.1) as the basis for the sequence alignments. General overviews of each component of the database construction, along with URLs pointing to the specific workflows with the Galaxy toolshed, are outlined below. Upon completion of the three workflows for each RNASeq sample, the records from the three pipelines were concatenated to create a completed sample-specific search database for each of the 10 animals in the study.

\section{Single amino acid variant (SAV) database construction and workflow}

Within the SAV workflow, RNA-Seq reads from one sample are aligned to the vervet reference genome using Tophat [33], single nucleotide variant calls are made using SAMtools [34], and the subset of identified SNPs which reside within exons are subsequently annotated using SnpEff [35]. A tool developed within Galaxy-P called "SNPeff to Peptide Fasta" is used to convert the nucleotide sequences into the expected corresponding amino acid sequences. The complete workflow can be found here: http://toolshed.g2.bx.psu.edu/view/galaxyp/ proteomics_rnaseq_sap_db_workflow.

\section{Splice junction (SJ) database construction and workflow}

The SJ workflow begins by aligning the RNA-Seq reads to the reference vervet genome as well as the Ensembl gene models for the species. The coordinates of all the detected junctions are compared between the two, and only those junctions mapping to the reference genome but not the Ensembl gene model are retained for the SJ annotation. The Galaxy-P program "Translate BED sequences" is used to convert the SIs identified by the RNA-Seq reads into the corresponding polypeptide sequences. Full details are available here: http://toolshed.g2.bx.psu.edu/view/galaxyp/ proteomics_rnaseq_splice_db_workflow. 
Transcript abundance-based database reduction workflow In order to reduce the records of proteins based on transcript abundance, RNA-Seq data is quantified by RSEM [36] within the Galaxy-P framework. Quantitative values are normalized and output in transcripts per million (TPM). Text manipulation tools in Galaxy concatenate the protein FASTA data with the transcript identifiers and TPM values, and all records where the values are less than one TPM are excluded from the search database, in accordance with our standard RNA-Seq quality control procedures. Including transcripts with lower abundance increases false-positive alignments, and would require validation through deep sequencing to confirm the presence of the transcript. The workflow repository with the Galaxy toolshed is listed here: http://toolshed.g2.bx.psu.edu/view/ galaxyp/proteomics_rnaseq_reduced_db_workflow.

\section{MS-based proteomics}

Proteins were extracted from liver tissue using RIPA lysis buffer, and separated on $4-12 \%$ gradient Bis-Tris gel. Three gel slices were excised and each was reduced with $10 \mathrm{mM}$ DTT for $30 \mathrm{~min}$ at room temperature and alkylated with $55 \mathrm{mM}$ iodoacetamide in $100 \mathrm{mM}$ ammonium bicarbonate for $30 \mathrm{~min}$ at room temperature. The gel pieces were subsequently washed with ultrapure $100 \mathrm{mM}$ ammonium bicarbonate, dehydrated with 100\% acetonitrile, and dried by Speedvac for 2-3 min.

Samples were then digested with trypsin (Promega, V5280) at $37{ }^{\circ} \mathrm{C}$ overnight. Formic acid (1\%) was added to the trypsinized samples to quench the proteolysis, and the peptides were desalted and concentrated using $C_{18}$ ZipTips (Millipore, Z720046-960EA). HPLC separation was performed on a $15 \mathrm{~cm}$ column of $3 \mu \mathrm{m}$ diameter which was packed in house with $\mathrm{C}_{18}$ beads. Peptides were loaded onto the column at a flow rate of $400 \mathrm{nl} / \mathrm{min}$ for $3 \mathrm{~h}$ and MS data were acquired by a data dependent scanning on the Thermo Scientific Orbitrap Elite mass spectrometer utilizing a default top 15 method.

Raw mass spectrometry (MS) files were subsequently analyzed in the program Morpheus [21]. The following settings were used in all searches: Assumed Precursor Charge States, Minimum $=2$; Assumed Precursor Charge States, Maximum $=4$; MS $/$ MS Peak Filtering, Maximum Number of Peaks $=400 ;$ MS $/$ MS Analysis, Assign Charge States $=$ enabled; Protease $=$ trypsin (no proline rule); Maximum Missed Cleavages = 2; Initiator Methionine Behavior = variable; Fixed Modifications $=$ carbamidomethylation of $\mathrm{C}$; Variable Modifications = oxidation of $\mathrm{M}$; Maximum Variable Modification Isoforms Per Peptide $=1024$; Precursor Mass Tolerance $= \pm 2.1 \mathrm{Da}$ (monoisotopic); Precursor Monoisotopic Peak Correction $=$ disabled; Product Mass Tolerance $= \pm 0.025 \mathrm{Da}$ (monoisotopic); Maximum False Discovery Rate $=1 \%$.
For each liver sample, two sets of Morpheus output files were created; the first analysis was searched using the reference vervet monkey database and the second analysis was searched utilizing the sample-specific database created by the Galaxy-P pipelines described above.

\section{Comparative proteomic analyses}

Prior to comparison of the proteomic results, the six sets of output files from the Morpheus program for each of the liver samples ( 3 fractions per sample, run against 2 search databases $=6$ files/sample) were combined and transformed to create unique identifiers for all of the peptide spectral match records. This permits the direct comparison of spectra matched from the raw MS files. The search database file size comparisons and wall clock times were extracted from the Morpheus summary files. The "VennDiagram" package in R (https://CRAN.R-project.org/package=VennDiagram) was used to create the lists of unique peptides and protein groups, as well as Venn diagram image files. An $\mathrm{R}$ markdown document outlining the tidying and concatenation of the Morpheus output files, along with the creation of the Venn diagrams, can be found in Additional file 1. Gene set enrichment analyses were conducted to identify classes of proteins overrepresented within the list of proteins identified by the reference database but not the samplespecific databases $[37,38]$.

\section{Results}

Search databases curated from RNA-Seq data are smaller and computationally more efficient than reference genome databases

To demonstrate the utility of RNA-Seq derived proteomics search databases, we created sample-specific databases (SSdb) for each liver sample from 10 different vervet monkeys based on sequenced mRNA extracted from the same tissue sample as the protein being analyzed by MS. As outlined above, this procedure creates a unique optimized search database for each sample from the RNA-Seq data, and each MS dataset for a given sample is searched against just the SSdb. For each of the 10 samples, the peptide spectral matching performance was compared between the SSdb and a search database created from the reference vervet genome (REFdb). The descriptive statistics for the RNA-Seq alignments and MS/MS raw data are outlined in Table 1. The RNA-Seq read depth ranged from 6.5 million to 10.5 million mapped reads for the 10 samples. Despite this variability, we found no relationship between RNA-Seq read depth and peptide spectral matches (PSMs) or unique peptides identified in the samples when searched by the SSdb.

Restricting the size of SSdb to transcripts with an abundance of 1 TPM or more condensed the search database size and Morpheus compute time when 
Table 1 Descriptive statistics for the RNA-Seq and mass spectrometry analyses utilizing the Vervet reference search database (REFdb, 19,255 gene entries) and the sample-specific databases (SSdb)

\begin{tabular}{|c|c|c|c|c|c|c|c|c|c|}
\hline \multirow[t]{2}{*}{ Sample } & \multicolumn{2}{|c|}{ RNA-Seq } & \multicolumn{2}{|c|}{ SSdb Entries } & \multirow[t]{2}{*}{ Mass Spectra } & \multicolumn{2}{|c|}{ PSMs } & \multicolumn{2}{|c|}{ Peptide IDs } \\
\hline & RNA-Seq reads & $\%$ reads aligned & Genes & Novel SJs & & REFdb & $\mathrm{SSdb}$ & REFdb & $\mathrm{SSdb}$ \\
\hline 1030 & $7,040,525$ & 55.5 & 13,804 & 4069 & 80,003 & 26,525 & 26,680 & 9765 & 9702 \\
\hline 1211 & $6,585,341$ & 68.8 & 15,782 & 7171 & 79,381 & 27,288 & 27,673 & 10,532 & 10,527 \\
\hline 1238 & $6,594,936$ & 67.1 & 15,659 & 6595 & 78,444 & 19,600 & 19,898 & 9349 & 9354 \\
\hline 1245 & $6,730,432$ & 64.0 & 13,901 & 4089 & 80,281 & 29,143 & 29,193 & 10,503 & 10,463 \\
\hline 1248 & $10,504,974$ & 69.4 & 15,513 & 7429 & 80,221 & 22,205 & 22,479 & 9120 & 9162 \\
\hline 1254 & $9,127,588$ & 62.5 & 15,936 & 7641 & 79,675 & 23,655 & 23,738 & 9334 & 9385 \\
\hline 1291 & $6,575,182$ & 67.9 & 13,354 & 3653 & 79,960 & 30,623 & 30,722 & 11,478 & 11,652 \\
\hline 1347 & $6,637,842$ & 56.6 & 13,284 & 3147 & 78,791 & 17,284 & 17,037 & 8633 & 8575 \\
\hline 1448 & $8,019,158$ & 65.0 & 15,668 & 6419 & 71,853 & 15,612 & 15,561 & 7582 & 7593 \\
\hline 1467 & $9,983,615$ & 66.2 & 16,176 & 7305 & 78,781 & 20,101 & 20,162 & 9177 & 9223 \\
\hline
\end{tabular}

compared to the REFdb. On average, the SSdbs were $77 \%$ of the size of the REFdb, and compute times in Morpheus averaged 53\% faster for SSdbs compared to the REFdb (defined by ([REFdb time/SSdb time]-1)).

Interestingly, for two samples (samples 1347 and 1448), the search against the REFdb resulted in more peptide spectral matches (aon average $0.6 \%$ ) when compared to the search using the SSdb. Similarly, for three samples (samples 1030, 1245, and 1347) the analysis against the REFdb identified slightly more peptides compared to the search using the SSdb (on average 0.5\%). Given the lower number of RNA-Seq reads or mass spectra obtained for some of these samples, it is conceivable that this difference is due to variation in sample preparation or sample quality. Partial degradation of tissue samples would affect both RNA and protein recovery, and may have impacted the analyses presented here.

\section{RNA-Seq derived search databases identify peptides not annotated in the vervet reference genome}

Next, we combined the search results across the 10 samples to compare the unique peptides and protein groups identified in vervet monkey liver samples by the REFdb versus the SSdb. These results are shown in the Venn diagram of Fig. 1. We identified 601 peptides in analyses using the SSdb that were not identified using the REFdb.

The first set of these peptides represents peptides that match newly identified SJs not annotated in the gene models of the reference genome. Of the 284 SJ peptide search records identified and annotated from the RNASeq data, we identified 47 peptides by MS in more than one sample, which suggests these matches represent incompletely annotated genes of the vervet genome. Consequently, the results of these proteomics analyses could aid in the improved annotation of the gene models. Another 45 peptides mapped to a single sample, bringing the total number of distinctly SJ-mapped peptides identified in these samples to 92. A comparative analysis of these peptide sequences with other primates utilizing BLASTP revealed that the majority of the identified peptides (53/92) could be matched with an orthologous protein [39]. The complete catalogue of SJs with their corresponding peptides can be found in Additional file 2 .

A second set of peptides uniquely identified in searches using the SSdb are peptides that map to an SAV record where the amino acid variant resides within the predicted tryptic peptide that matched in the search. In total, 192 peptide matches representing 101 distinct peptides were found in the 10 samples by the samplespecific analyses using the SSdb. Of these 101 distinct peptides that conformed SAVs identified in the RNA-Seq data, 37 peptides were identified in more than one sample. A list of all peptides matching SAV records are included in Additional file 2.

These first two categories of peptides identified within the SSdb analyses represent search results we expected to obtain from the RNA-Seq-based proteogenomic approach because the mRNA read data create search records which accurately predict the respective peptide fragments seen in the MS dataset.

\section{Reduction of the search database size recovers peptide identifications}

While we might expect the same number of matches to reference protein entries contained in both the SSdb and REFdb searches, a total of 313 peptides were identified as matches to reference proteins in the search against the SSdb but not the REFdb. Morpheus, like many other spectral matching algorithms, uses a decoy-based searching approach to empirically estimate and maintain a $1 \%$ false discovery rate (FDR). The inclusion of these peptides may result from the adjustment of the absolute FDR that arises from the reduction in the absolute size of the SSdb compared to the REFdb. This is supported 
Sample Specific Database vs Reference Database Proteins Identified

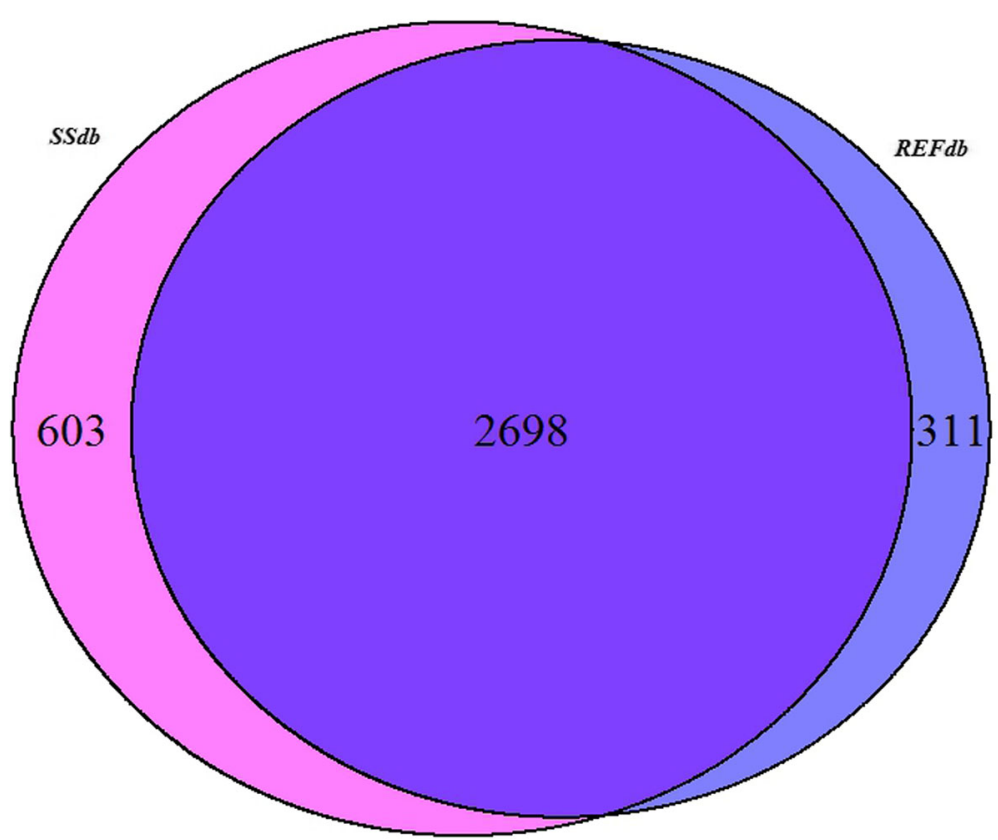

Fig. 1 Sample specific database vs reference database proteins identified

by the fact that the q-values of this subset of SSdbidentified peptides is higher, on average, compared to the overall average q-value of all peptides identified using the SSdb search. Of the "reference" peptides identified using the SSdb, only $16 \%$ (151 of 948) were seen in two or more samples. The peptides identified exclusively in the SSdb analyses are listed in Additional file 3.

\section{Peptide identifications missed by RNA-Seq-derived data- bases are predominantly structural proteins}

While the minimization of the search database helps recover some true positive matches, as described above, concerns about restricting proteome search databases based upon RNA-Seq data could arise when a protein's abundance is poorly correlated with its corresponding transcript abundance, or when proteins might be derived from a tissue of origin different from which they currently reside. Extracellular structural proteins or chromatin-associated proteins with long-half-lives would be likely candidates for the former category, while growth factors, cytokines or contaminating proteins might comprise the latter. We surveyed the list of peptides identified by REFdb but not SSdb analyses and identified 1950 total matches mapping to 891 unique peptides, of which 506 (57\%) were identified in 2 or more liver samples. Those 506 peptides correspond to 238 unique protein entries in the vervet ENSEMBL database. Gene set enrichment analysis revealed that this list of proteins was indeed overrepresented by structural, cytoskeletal, and ribonucleotide binding proteins. Table 2 lists the significantly enriched categories identified in this subset of proteins. The peptides identified by the REFdb but not the SSdbs searches can be found in Additional file 4.

Though distinct differences in the peptides identified by the SSdb versus REFdb have been revealed by this comparative exercise, it is perhaps equally important to highlight that SSdb analyses are capable of matching nearly 98\% of the same spectra as the REFdb. The customized databases created from RNA-Seq robustly identify proteins from tissue samples, identify peptide variation that would otherwise be missed by searching against the reference annotation, and perform these functions using smaller file sizes and faster compute times.

\section{Discussion}

This work represents the implementation and proof-ofconcept application of an established RNA-Seq proteogenomic approach to improve the identification of proteins in non-human primate proteomics experiments, such as the vervet monkey liver sample analyses reported here. Several non-human primate genomes have been drafted but incompletely annotated because a limited number of animals have been sequenced. Less common sequence and splice variants will continue to be incorporated as DNA sequencing sample sizes 
Table 2 Gene Set Enrichment Analysis for proteins identified by reference but not sample-specific databases

\begin{tabular}{|c|c|c|c|}
\hline GO Annotation & Description & $p$-value & FDR q-value \\
\hline GO: STRUCTURAL MOLECULE ACTIVITY & $\begin{array}{l}\text { The action of a molecule that contributes to the structural integrity of a complex } \\
\text { or assembly within or outside a cell. }\end{array}$ & $3.03 \times 10^{-15}$ & $3.42 \times 10^{-11}$ \\
\hline GO: OXIDATION REDUCTION PROCESS & $\begin{array}{l}\text { A metabolic process that results in the removal or addition of one or more } \\
\text { electrons to or from a substance, with or without the concomitant removal } \\
\text { or addition of a proton or protons. }\end{array}$ & $9.8 \times 10^{-14}$ & $5.52 \times 10^{-10}$ \\
\hline GO: DNA PACKAGING COMPLEX & A protein complex that plays a role in the process of DNA packaging. & $7.61 \times 10^{-13}$ & $2.86 \times 10^{-9}$ \\
\hline GO: EXTRACELLULAR SPACE & $\begin{array}{l}\text { That part of a multicellular organism outside the cells proper, usually taken } \\
\text { to be outside the plasma membranes, and occupied by fluid. }\end{array}$ & $1.25 \times 10^{-11}$ & $2.81 \times 10^{-8}$ \\
\hline GO: PROTEIN DNA COMPLEX & A macromolecular complex containing both protein and DNA molecules. & $6.04 \times 10^{-11}$ & $1.13 \times 10^{-7}$ \\
\hline
\end{tabular}

increase and more complete transcriptional profiles across tissue types are reported. In the meantime, a proteogenomic approach should improve protein identification in MS experiments in these species, and may offer robust and reliable data to improve the existing annotation using both transcript and protein data. We demonstrated that this analysis approach, implemented in Galaxy-P, is capable of identifying peptides derived from unannotated splice junctions and non-synonymous coding substitutions revealed from the RNA-Seq read data. These peptides would have gone unmatched by searching the MS data using the vervet reference genome annotation data. These novel peptides likely represent a combination of common, yet previously unannotated gene isoforms as well as isoforms and variants private to individual animals studied.

As with other "omics" scale analyses, the iterative search process of shotgun proteomics presents challenges in balancing type I and type II error rates. The optimal search database would incorporate only those potential proteins which are likely to be found within a given sample; however, if the repertoire of proteins were already known, it would obviate the need for conducting proteomics experiments in the first place. Instead, RNASeq data can facilitate a compromise in the database curation process by predicting non-reference protein isoforms for inclusion while also utilizing information about transcript abundance to exclude individual gene sequences from the search records as the corresponding mRNA are not expressed and therefore the translated protein (or a peptide thereof) is unlikely to be identified by MS. While RNA-Seq data can be a useful benchmark for restricting the size of the MS search database, it should be noted that certain exceptions to the database exclusion process should be considered. Examples include proteins whose tissue of origin is different from the sampled tissue, such as blood-derived albumin, immunoglobulins, and complement proteins, or longlived structural proteins such as collagen or ribosomal proteins, whose protein abundance is uncoupled from their corresponding mRNA expression in the tissue of interest at the time of sample collection. By comparing peptide matches made using RNA-Seq derived databases to peptides identified using the reference database, we have revealed a list of proteins routinely found in vervet livers that do not have corresponding mRNA abundance levels from RNA-Seq read data. Including these protein records in the construction of sample-specific databases for liver samples could mitigate the loss of information in future proteomics experiments.

The reduction of the search database size and the included protein records significantly impacts the confidence with which peptides and proteins are identified. Due to the smaller number of records to compare an experimental spectrum to, the confidence with which individual peptides are assigned to the correct record is higher. However, these "improved" matches have another consequence: some experimental peptide spectra may not be confidently assigned to a specific sequence in the REFdb analysis since the match may not fall under the stringent $1 \%$ FDR commonly required to confirm a match. However, using the smaller SSdb, the same match is now made with a FDR of less than $1 \%$ (simply based on the smaller number of searches), resulting in some additional "reference" proteins being identified in the SSdb searches at a 1\% FDR but not in the REFdb searches. It is possible that these matches may include some low confidence peptide matches, but overall these additional peptide matches emphasize the additional power that is gained from reducing the search database to only relevant records of expressed proteins and peptide sequences.

Our analysis only used one standard established analysis approach for these comparisons. The analysis pipeline implemented in Galaxy includes the Morpheus search algorithm which empirically calculates the FDR for peptide matches obtained in the search. Numerous other approaches have been proposed for the analysis of proteogenomic data, and the assessment of FDR in peptide and protein identifications. It is likely that some of these approaches, such as analyzing FDR separately for REFdb matches and matches to novel SSdb records derived from RNA-Seq [19] or alternative programs to calculate FDR in these datasets [40-42], would improve 
the results presented here, and further enhance the utility of this proteogenomic approach for non-human primate proteomics. However, a detailed comparison of these different analysis approaches was not the goal of the current study, and future studies will help define the optimal approach for a proteogenomic analysis in these species, including the optimal RNA-Seq coverage and the depth of proteomic analysis. Prior analyses have generated far more detailed mass spectral analysis data (500,000 mass spectra compared to 80,000 used in our study), and it remains to be seen what the optimal approach will be [16]. As proteogenomic approaches continue to gain momentum in shotgun proteomics experiments, we anticipate further refinement of search databases to account for biochemical variability in peptides which arise from post-translational modifications (PTMs). A recent publication has outlined an approach to parsimoniously account for peptide mass shifts caused by PTMs through incorporating Uniprot annotation data $([43,44])$. Similarly, proteogenomics can incorporate findings from complimentary NGS approaches, such as ribosomal profiling, to expand the prediction of the protein-coding products from novel coding sequences [45] and IncRNA molecules previously presumed to be untranslated $[44,46]$. Continued refinements to search databases and proteomics search algorithms will accelerate the accurate identification and quantification of peptides in MS analyses, and it will complement and improve the genome annotation of animal research model organisms and help researchers utilize shotgun proteomics to implicate protein changes associated with pathophysiologic processes. Ultimately, the proteomic validation of novel splice variants and non-synonymous sequence variants will greatly enhance the ongoing efforts of genome annotation, especially in model species with poorly annotated genomes, such as many non-human primates.

\section{Conclusions}

A proteogenomic approach to the analysis of liver shotgun proteomic data from a nonhuman primate species, the vervet/African green monkey (Chlorocebus aethiops sabaeus), demonstrates that the use of sample-derived RNA-Seq data, as anticipated, improves peptide identification and the accuracy and confidence of protein identification, while simultaneously reducing the search database space and the resulting computing effort required for the data analysis. Novel peptides including sequence variants identified by RNA-Seq, as well as new splice variants uncovered in the transcriptional analysis account for the majority of the novel peptides identified, highlighting the importance of proteogenomic approaches in species with limited available genome sequence data and gene annotation, such as non-human primates.

\section{Additional files}

Additional file 1: Outlining the data transformations and analyses of the Morpheus file outputs. (HTML $641 \mathrm{~kb}$ )

Additional file 2: Listing the SAV and SJ derived peptides identified exclusively by the sample-specific databases. (XLSX 46 kb)

Additional file 3: Listing the reference genome peptides identified exclusively by the sample-specific databases. (XLS 343 kb)

Additional file 4: Listing the peptides identified by the REFdb but not the SSdb searches. (XLS $541 \mathrm{~kb}$ )

\begin{abstract}
Abbreviations
AIDS: Acquired immune deficiency syndrome; DTT: Dithiothreitol; FDR: False discovery rate; GO: Gene ontology; GSEA: Gene set enrichment analysis; HPLC: High performance liquid chromatography; IncRNA: Long, non-coding ribonucleic acid; mRNA: Messenger ribonucleic acid; MS: Mass spectrometry; PSM: Peptide spectral match; REFdb: Reference database; SAV: Single amino variant; SIV: Simian immunodeficiency virus; SJ: Splice junction; SNP: Single nucleotide polymorphism; SSDb: Sample-specific database; TPM: Transcripts per million
\end{abstract}

\section{Acknowledgements}

Not applicable

\section{Funding}

Animal sample collection supported by P40 OD010965 and UL1 TR004120 (KK) Proteogenomic analyses and interpretation supported by NIGMS R01 GM109099 (MO and LMS). AJC supported by the CIBM Training Program 5T15LM007359. The authors also acknowledge grant support from NIH Grant P51 OD011133 and facilities support from NIH Research Facilities Improvement Program Grants C06 RR 1 C06 RR013556 and 1 C06 RR017515. The funding agencies did not have any role in the design of the study, collection, analysis, and interpretation of data, or in writing the manuscript.

\section{Availability of data and materials}

The datasets used and analyzed during the current study are available from the corresponding author on reasonable request.

\section{Authors' contributions}

JMP, MO, LAC, and KK conceived the study. KK oversaw the selection and sampling of the vervet liver biopies. LAC and JG generated the RNA-Seq data. MO and AJ generated the proteomics data. LMS, MRS, AJC, and JG contributed to the construction of the sample-specific databases. JMP analyzed the mass spectrometry data and conducted the comparative analyses. JMP and MO drafted the manuscript, and LMS, AJC, and LAC provided significant editorial revisions. All authors have read and approved of the final version of the manuscript.

Ethics approval and consent to participate

All animal procedures were approved by the Wake Forest School of Medicine Clinical and Translational Science Institute Institutional Animal Care and Use Committee and conducted in AAALAC approved facilities. All animals included in this study were female vervet/African green monkeys (Chlorocebus aethiops sabaeus) from the Vervet Research Colony (VRC) at Wake Forest School of Medicine. All monkeys were US-colony born within the VRC, which is a multi-generational, pedigreed, and genotyped colony originally founded in 1975 by the University of California Los Angeles, with 57 animals imported from St. Kitts and Nevis. In early 2008, the VRC was transferred to Wake Forest School of Medicine and remains a continuously $\mathrm{NIH}$-supported national research resource.

Consent for publication

Not applicable

Competing interests

The authors declare that they have no competing interests. 


\section{Publisher's Note}

Springer Nature remains neutral with regard to jurisdictional claims in published maps and institutional affiliations.

\begin{abstract}
Author details
'Department of Genetics, Texas Biomedical Research Institute, San Antonio, Texas, USA. ${ }^{2}$ Department of Chemistry, University of Wisconsin, Madison, Wisconsin, USA. ${ }^{3}$ Genome Center of Wisconsin, University of Wisconsin, Madison, Wisconsin, USA. ${ }^{4}$ Department of Pathology and Comparative Medicine, Wake Forest School of Medicine, Winston-Salem, North Carolina, USA. ${ }^{5}$ Southwest National Primate Research Center, Texas Biomedical Research Institute, San Antonio, Texas, USA. ${ }^{6}$ Current address: Department of Internal Medicine, Section of Molecular Medicine, Wake Forest School of Medicine, NRC Building, G-55, Winston-Salem, North Carolina 27157, USA.
\end{abstract}

Received: 7 March 2017 Accepted: 3 November 2017

Published online: 13 November 2017

\section{References}

1. Heunis T, Dippenaar A, Warren RM, van Helden PD, van der Merwe RG, Gey van Pittius NC, Pain A, Sampson SL, Tabb DL. Proteogenomic investigation of strain variation in clinical mycobacterium tuberculosis isolates. J Proteome Res. 2017; doi: 10.1021/acs.jproteome.7b00483.

2. Swearingen KE, Lindner SE, Flannery EL, Vaughan AM, Morrison RD, Patrapuvich R, Koepfli C, Muller I, Jex A, Moritz RL, Kappe SHI, Sattabongkot J, Mikolajczak SA. Proteogenomic analysis of the total and surface-exposed proteomes of plasmodium vivax salivary gland sporozoites. PLoS Negl Trop Dis. 2017;11(7):e0005791.

3. Krishna R, Xia D, Sanderson S, Shanmugasundram A, Vermont S, Berna A, Daniel-Naguib G, Ghali F, Brunk BP, Roos DS, Wastling JM, Jones AR. A large-scale proteogenomics study of apicomplexan pathogensToxoplasma gondii and Neospora caninum. Proteomics. 2015;15(15): 2618-28.

4. Zhu FY, Chen MX, Ye NH, Shi L, Ma KL, Yang JF, Cao YY, Zhang Y, Yoshida T, Fernie AR, Fan GY, Wen B, Zhou R, Liu TY, Fan T, Gao B, Zhang D, Hao GF, Xiao S, Liu YG, Zhang J. Proteogenomic analysis reveals alternative splicing and translation as part of the abscisic acid response in Arabidopsis seedlings. Plant J. 2017;91(3):518-33.

5. Grossmann J, Fernández H, Chaubey PM, Valdés AE, Gagliardini V, Cañal MJ, Russo G, Grossniklaus U. Proteogenomic analysis greatly expands the identification of proteins related to reproduction in the Apogamous Fern Dryopteris Affinis Ssp. Affinis. Front Plant Sci. 2017;8:336.

6. Zargar SM, Mahajan R, Nazir M, Nagar P, Kim ST, Rai V, Masi A, Ahmad SM, Shah RA, Ganai NA, Agrawal GK, Rakwal R. Common bean proteomics: present status and future strategies. J Proteome. 2017;169:233-38. doi:10.1016/j.jprot.2017.04.010.

7. Bryant L, Patole C, Cramer R. Proteomic analysis of the medicinal plant Artemisia Annua: data from leaf and trichome extracts. Data Brief. 2016:7:325-31.

8. Trapp J, Gaillard JC, Chaumot A, Geffard O, Pible O, Armengaud J. Ovary and embryo proteogenomic dataset revealing diversity of vitellogenins in the crustacean Gammarus fossarum. Data Brief. 2016;8:1259-62.

9. Caruana NJ, Cooke IR, Faou P, Finn J, Hall NE, Norman M, Pineda SS, Strugnell JM. A combined proteomic and transcriptomic analysis of slime secreted by the southern bottletail squid, Sepiadarium Austrinum (Cephalopoda). J Proteome. 2016;148:170-82.

10. Whitelaw BL, Strugnell JM, Faou P, da Fonseca RR, Hall NE, Norman M, Finn J, Cooke IR. Combined Transcriptomic and proteomic analysis of the posterior salivary gland from the southern blue-ringed octopus and the southern sand octopus. J Proteome Res. 2016;15(9):3284-97.

11. McAfee A, Harpur BA, Michaud S, Beavis RC, Kent CF, Zayed A, Foster L. Toward an upgraded honey bee (Apis Mellifera L.) genome annotation using Proteogenomics. J Proteome Res. 2016;15(2):411-21.

12. Bottje WG, Lassiter K, Piekarski-Welsher A, Dridi S, Reverter A, Hudson NJ, Kong BW. Proteogenomics reveals enriched ribosome assembly and protein translation in Pectoralis major of high feed efficiency pedigree broiler males. Front Physiol. 2017:8:306.

13. Anderson KJ, Vermillion KL, Jagtap P, Johnson JE, Griffin TJ, Andrews MT. Proteogenomic analysis of a hibernating mammal indicates contribution of skeletal muscle physiology to the hibernation phenotype. J Proteome Res. 2016;15(4):1253-61.
14. Marx H, Hahne H, Ulbrich SE, Schnieke A, Rottmann O, Frishman D, Kuster B. Annotation of the domestic pig genome by quantitative Proteogenomics. J Proteome Res. 2017;16(8):2887-98.

15. Chemonges S, Gupta R, Mills PC, Kopp SR, Sadowski P. Characterisation of the circulating acellular proteome of healthy sheep using LC-MS/MS-based proteomics analysis of serum. Proteome Sci. 2017;15:11.

16. Sheynkman GM, Johnson JE, Jagtap PD, Shortreed MR, Onsongo G, Frey BL, et al. Using galaxy-P to leverage RNA-Seq for the discovery of novel protein variations. BMC Genomics. 2014;15:703.

17. Wen B, Xu S, Sheynkman GM, Feng Q, Lin L, Wang Q, et al. sapFinder: an R/ bioconductor package for detection of variant peptides in shotgun proteomics experiments. Bioinformatics. 2014;30(21):3136-8.

18. Wen B, Xu S, Zhou R, Zhang B, Wang X, Liu X, et al. PGA: an R/bioconductor package for identification of novel peptides using a customized database derived from RNA-Seq. BMC Bioinformatics. 2016:17(1):244.

19. Nesvizhskii Al. Proteogenomics: concepts, applications and computational strategies. Nat Methods. 2014;11(11):1114-25.

20. Afgan E, Baker D, van den Beek M, Blankenberg D, Bouvier D, Čech M, et al. The galaxy platform for accessible, reproducible and collaborative biomedical analyses: 2016 update. Nucleic Acids Res. 2016;44(W1):W3-W10.

21. Wenger $C D$, Coon JJ. A proteomics search algorithm specifically designed for high-resolution tandem mass spectra. J Proteome Res. 2013;12(3):1377-86.

22. Schmitz JE, Zahn RC, Brown CR, Rett MD, Li M, Tang H, et al. Inhibition of adaptive immune responses leads to a fatal clinical outcome in SIV-infected pigtailed macaques but not vervet African green monkeys. PLoS Pathog. 2009;5(12):e1000691.

23. Zahn RC, Rett MD, Korioth-Schmitz B, Sun Y, Buzby AP, Goldstein S, et al. Simian immunodeficiency virus (SIV)-specific CD8+ T-cell responses in vervet African green monkeys chronically infected with SIVagm. J Virol. 2008:82(23):11577-88

24. Burke MW, Ptito M, Ervin FR, Palmour RM. Hippocampal neuron populations are reduced in vervet monkeys with fetal alcohol exposure. Dev Psychobiol. 2015;57(4):470-85

25. Bouskila J, Harrar V, Javadi P, Beierschmitt A, Palmour R, Casanova C, et al Cannabinoid receptors CB1 and CB2 modulate the Electroretinographic waves in Vervet monkeys. Neural Plast. 2016;2016:1253245.

26. Jasinska AJ, Schmitt CA, Service SK, Cantor RM, Dewar K, Jentsch JD, et al. Systems biology of the vervet monkey. ILAR J. 2013;54(2):122-43.

27. Voruganti VS, Jorgensen MJ, Kaplan JR, Kavanagh K, Rudel LL, Temel R, et al. Significant genotype by diet $(G \times D)$ interaction effects on cardiometabolic responses to a pedigree-wide, dietary challenge in vervet monkeys (Chlorocebus Aethiops Sabaeus). Am J Primatol. 2013;75(5):491-9.

28. Kavanagh K, Flynn DM, Nelson C, Zhang L, Wagner JD. Characterization and validation of a streptozotocin-induced diabetes model in the vervet monkey. J Pharmacol Toxicol Methods. 2011;63(3):296-303.

29. Kavanagh K, Fairbanks LA, Bailey JN, Jorgensen MJ, Wilson M, Zhang L, et al. Characterization and heritability of obesity and associated risk factors in vervet monkeys. Obesity (Silver Spring). 2007;15(7):1666-74

30. Warren WC, Jasinska AJ, García-Pérez R, Svardal H, Tomlinson C, Rocchi M, et al. The genome of the vervet (Chlorocebus Aethiops Sabaeus). Genome Res. 2015;25(12):1921-33.

31. Jagtap PD, Johnson JE, Onsongo G, Sadler FW, Murray K, Wang Y, et al. Flexible and accessible workflows for improved proteogenomic analysis using the galaxy framework. J Proteome Res. 2014;13(12):5898-908.

32. Sheynkman GM, Shortreed MR, Frey BL, Smith LM. Discovery and mass spectrometric analysis of novel splice-junction peptides using RNA-Seq. Mol Cell Proteomics. 2013;12(8):2341-53.

33. Trapnell C, Pachter L, Salzberg SL. TopHat: discovering splice junctions with RNA-Seq. Bioinformatics. 2009;25(9):1105-11.

34. Li H. A statistical framework for SNP calling, mutation discovery, association mapping and population genetical parameter estimation from sequencing data. Bioinformatics. 2011;27(21):2987-93.

35. Cingolani P, Platts A, Wang LEL, Coon M, Nguyen T, Wang L, et al. A program for annotating and predicting the effects of single nucleotide polymorphisms, SnpEff: SNPs in the genome of Drosophila Melanogaster strain w1118; iso-2; iso-3. Fly (Austin). 2012;6(2):80-92.

36. Li B, Dewey CN. RSEM: accurate transcript quantification from RNASeq data with or without a reference genome. BMC Bioinformatics. 2011;12:323.

37. Subramanian A, Tamayo P, Mootha VK, Mukherjee S, Ebert BL, Gillette MA et al. Gene set enrichment analysis: a knowledge-based approach for 
interpreting genome-wide expression profiles. Proc Natl Acad Sci U S A. 2005;102(43):15545-50

38. Gene Ontology Consortium. Gene ontology consortium: going forward. Nucleic Acids Res. 2015;43(Database issue):D1049-56.

39. Altschul SF, Madden TL, Schäffer AA, Zhang J, Zhang Z, Miller W, et al. Gapped BLAST and PSI-BLAST: a new generation of protein database search programs. Nucleic Acids Res. 1997;25(17):3389-402.

40. Wang X, Slebos RJ, Wang D, Halvey PJ, Tabb DL, Liebler DC, Zhang B. Protein identification using customized protein sequence databases derived from RNA-Seq data. J Proteome Res. 2012;1 1(2):1009-17.

41. Li H, Park J, Kim H, Hwang KB, Paek E. Systematic comparison of falsediscovery-rate-controlling strategies for Proteogenomic search using spikein experiments. J Proteome Res. 2017;16(6):2231-9.

42. Ma C, Xu S, Liu G, Liu X, Xu X, Wen B, Liu S. Improvement of peptide identification with considering the abundance of mRNA and peptide. BMC Bioinformatics. 2017;18(1):109.

43. Cesnik AJ, Shortreed MR, Sheynkman GM, Frey BL, Smith LM. Human proteomic variation revealed by combining RNA-Seq Proteogenomics and global post-translational modification (G-PTM) search strategy. J Proteome Res. 2016;15(3):800-8.

44. Ingolia NT, Lareau LF, Weissman JS. Ribosome profiling of mouse embryonic stem cells reveals the complexity and dynamics of mammalian proteomes. Cell. 2011;147(4):789-802.

45. Raj A, Wang SH, Shim H, Harpak A, Li YI, Engelmann B, et al. Thousands of novel translated open reading frames in humans inferred by ribosome footprint profiling. elife. 2016;27:5.

46. Ji Z, Song R, Regev A, Struhl K. Many IncRNAs, 5'UTRs, and pseudogenes are translated and some are likely to express functional proteins. elife. 2015;4:e08890.

\section{Submit your next manuscript to BioMed Central and we will help you at every step:}

- We accept pre-submission inquiries

- Our selector tool helps you to find the most relevant journal

- We provide round the clock customer support

- Convenient online submission

- Thorough peer review

- Inclusion in PubMed and all major indexing services

- Maximum visibility for your research

Submit your manuscript at www.biomedcentral.com/submit 